\title{
Capillary electrochromatography, electrically enhanced concentration, instrumentation of a chromatographic system for space station, and dynamic observation and analysis of human sweat
}

T. Tsuda

Nagoya Institute of Technology, Gokiso, Showa, Nagoya 466-8555, Japan

Features of capillary electrochromatography and related topics are described.

n our research group, we have the following projects:

(1) Capillary electrochromatography (CEC).

(2) Estimation of physical parameters by using CEC and capillary electrophoresis (CE).

(3) Developing the methodology of preconcentration under using electric field.

(4) Micro-separation system for the analysis of single cells and their inner fluid.

(5) Instrumentation of a chromatographic system in space station for on-site separation in the life science experiments.

(6) Dynamic observation of the secretion of human sweat, and the analysis of components included in it.

\section{Capillary electrochromatography}

We have started electrochromatography from really initial stage. Our first report was published in 1982, entitled "Open-tubular capillary liquid chromatography with electroosmosis flow using a UV detector" [1]. And then we named this method as electrochromatography. This word was firstly employed in our report of "Electrochromatography using high applied voltage", published in 1986 [2]. The word of capillary electrochromatography was also used in our report in 1994 [3].

We developed both capillary electrochromatography systems with and without pressurized flow [4,5]. We have recommended to use a liquid chromatographic pump (LC pump) in CEC or use an attachment to be able to press the inlet of column in the same level used in LC. There are following advantages we may count. (1) Column conditioning is performed very easily; (2) When you have a problem of bubble formation, you can exclude them by using an LC pump in short period; (3) When you are looking for the best chromatographic conditions, it is better to operate CEC with pressurized flow. You can survey the experimental conditions in a relatively short period. (4) When you would like to operate CEC without pressurized flow, you just stop the LC pump; (5) If you would like to use gradient mode, you can use just the LC device for gradient elution. From these advantages, we surely recommend to use a pump or have a same kind device when you run CEC.

In these days we have developed the instrumentation for CEC. These devices are summarized in the book, "Electric field applications in chromatography, industrial and chemical processes" [4].

In recent days we try to use ion exchange resin and cyclodextrin-modified supports in CEC. We have got the clear evidence that the equilibrium is effected by electric field.

\section{Physical phenomena under electric field}

From the relationship between electroosmotic flow velocity in a packed microcapillary column and $\mathrm{pH}$ of eluent, we estimated the dissociation constant of residual silanol group on the modified ODS silica gels [5]. Diffusion coefficients are estimated by the new method that a series of cycles of negative and positive voltage is applied for canceling the effect of flow profile by using open-tubular capillary columns [6]. Zone flow profiles of electroosmosis in both open-tubular and packed capillary columns are observed dynamically by using a microscopy. We find that the zone profile is reverse parabolic. The growth and decay of the electroosmotic flow due to the application and release of electric voltage, respectively, are very rapid, less than $1 / 15$ second [7]. Recently we find the equilibrium of a solute between mobile phase and ion exchange resin depends on the applied voltage. As this phenomenon is quite interesting, we are now under research to find out these kinds of experimental cases.

\section{Electrically enhanced preconcentration}

A pressurized flow and counter-migration due to electrophoretic mobility of a solute, can be used for sample 
concentration. An apparatus composed of two rooms with electrodes and connecting tubes is proposed [8]. We also developed an electroconcentration method using a small micro-syringe. This device is quite unique. It is possible to inject the concentrate directly to chromatography without changing vials [9].

\section{Proposal chromatographic system in space station}

In the near future a space station will be constructed under the international supports. In the station, Japan will have own laboratory. One of the big project in the space experiments is a field of life sciences. For supporting these experiments, it is necessary to obtain the experimental results in real time. Chromatographic system is one of the key instrumentation for this purpose. We are now planning to design the chromatographic system in space. The system should be used as liquid chromatography, capillary electrochromatography and capillary electrophoresis. We are now trying to construct this complex chromatographic system with the cooperation of Japan Space Agency. (NASDA).

\section{Dynamic observation of the secretion of human perspiration and the analysis of its component for getting the information of human body directly}

We have observed the sweat secretion on thumb, forehead, nose, etc. There are 200 to 300 sweat glands on the human thumb. By their dynamic microscopic monitoring, we find that the activities of the sweat glands were asynchronous when observed in unit periods of several seconds. The insensible and thermal perspirations are working 8 to 14 times per minute for controlling the temperature of human body. This is clearly recorded by using the instrument for measuring the perspiration volume with high sensitivity [10]. We recently found that the alcohol concentration in sweat is well correlated with the alcohol concentration in blood. By our proposed method, in which concurrent measurement of perspiration volume and absolute amount of alcohol are used, we can measure it every $5 \mathrm{~min}$. Therefore it may be possible to follow the process of digestion of alcohol in human body precisely.

\section{References}

1. Tsuda, T.; Nomura, K.; Nakagawa, G. J. Chromatogr. 1982, $248,241-247$

2. Tsuda, T. Anal. Chem. 1987, 59, 521-523.

3. Kitagawa, S.; Tsuda, T. J. Microcol. Sep. 1994, 6, 91-96.

4. Tsuda, T., Ed., Electric field Applications in Chromatography, industrial and Chemical Processes; VCH: Weinheim, 1995.

5. Kitagawa, S.; Tsuda, T. J. Microcol. Sep. 1995, 7, 59-64.

6. Tsuda, T.; Kitagawa, S.; Ono, T.; Maeda, M. J. Capillary Electrophoresis 1997, 4, 113-116.

7. Tsuda, T.; Kitagawa S.; Dadoo, R.; Zare, R.N. Bunseki Kagaku 1997, 46, 409-414.

8. Hori, A.; Matsumoto, T.; Nimura, Y.; Ikedo, M.; Okada, H.; Tsuda, T. Anal. Chem. 1993, 65, 2882-2886.

9. Kitagawa, S.; Tsuda, T.; Matsumoto, T. Bunseki Kagaku 1996, 45, 309-313.

10. Kamei, T.; Naitoh, K.; Nakashima, K.; Ohhashi, T.; Kitagawa, S.; Tsuda, T. J. Pharm. Biomed. Anal. 1997, 15, 1563-1569. 